\title{
Local for advanced, is this a paradox?
}

\author{
Maristella Bungaro, Chiara Paratore, Paolo Bironzo", Silvia Novello" \\ Medical Oncology Department, University of Turin, San Luigi Gonzaga Hospital, Orbassano, Italy \\ \#These authors contributed equally to this work. \\ Correspondence to: Dr. Chiara Paratore. Medical Oncology Department, San Luigi Gonzaga Hospital, Regione Gonzole 10, Orbassano 10043, Italy. \\ Email: ch.paratore@gmail.com.
}

Submitted Jun 21, 2020. Accepted for publication Mar 05, 2021.

doi: $10.21037 /$ tlcr-20-771

View this article at: http://dx.doi.org/10.21037/tlcr-20-771

Approximately $30 \%$ of patients with advanced non-small cell lung cancer (NSCLC) present with a limited number of metastatic sites at the time of diagnosis. This pattern, called 'oligometastatic', is commonly defined as an intermediate state between localized and widespread disease, according to the first definition proposed by Weichselbaum and Hellman in 1995 (1). They sensed that the reduced metastatic potential, the less aggressive biology and the limited number of lesions could offer a window for the use of local therapy on each active site of disease. Therefore, patients with oligometastatic disease might represent a unique subpopulation of advanced NSCLC, possibly amenable of curative intent treatments.

Accordingly, in the eighth edition of TNM classification, the $M$ descriptor was furtherly divided into three categories: M1a defining patients with contralateral pulmonary nodule(s) or pleural involvement without extrathoracic metastases, M1b for those with a single extrathoracic metastasis, and M1c in case of multiple extrathoracic metastases, even in a single organ. Such classification is based on the improved survival rates of patients with M1a/ M1b disease as compared to those with M1c (2).

Instead of being a unique entity, oligometastatic NSCLC can be further defined using a time-based classification. Indeed, while synchronous oligometastatic refers to patients whose primary tumor and a limited number of metastases occur at the same time, metachronous oligometastatic applies to clinical situations characterized by the appearance of metastases after the definitive treatment of the primary tumor. Finally, a third entity, named oligoprogressive disease, depicts those patients experiencing disease progression on a limited number of distant foci during systemic therapy, in the presence of a controlled primary tumor (3).
The increasing number of available loco-regional treatment strategies, especially minimally invasive surgery and stereotactic radiotherapy, have led to the introduction of synchronous oligometastatic NSCLC as a special treatment entity in the clinical practice guidelines by European Society of Medical Oncology from 2016 (4). However, even if acknowledging this entity was a step forward in research and daily practice, the optimal management of these patients is still far from being defined.

The first reason that made evidence left behind was the absence of a common definition. Consequently, clinical trials exploring loco-regional approaches in this disease entity are characterized by extreme heterogeneity regarding number of metastatic sites, type of involved organs, and staging procedures, leading to not comparable results.

For this reason, the European Organization for Research and Treatment of Cancer-Lung Cancer Group (EORTCLCG) started a consensus process to find a common definition of synchronous oligometastatic disease $(5,6)$. The work included a European survey, a systematic review and real-life case discussions. A subsequent consensus meeting established that the expression 'long-term disease control' should replace the term 'cure' in matter of disease treatment. For the definition of oligometastatic disease, a maximum number of 5 metastases in 3 different organs was proposed. This was based on a balance between the survey results (where the maximum number of lesions most frequently reported was 3 ) and those of the systematic review (where studies included patients with 1 to 8 distant metastases, and up to 5 sites in more than one third of trials). According to the EORTC-LCG consensus, all organs are potential oligometastatic sites, except diffuse serosal, meningeal and bone marrow involvement, as these sites cannot be treated with radical intent. Even if mediastinal lymph nodes 
are not considered as a metastatic site, a careful clinical staging of the $\mathrm{N}$ descriptor is of paramount, given the prognostic role of locoregional nodal involvement. However, histological confirmation of mediastinal involvement is limited to cases where management would change. 18F-FDG PET scan and brain imaging are mandatory in all cases. Finally, biopsy of a solitary metastatic site should be always performed, unless the risks related to the procedure are more than the benefits. Looking at survey results, overall survival (OS) was considered the best primary end-point when performing clinical trials in oligometastatic patients, even if that was the case of only one of the available randomized phase 2 studies (6).

The OligoCare project was then launched by EORTC and the European Society of Radiotherapy and Oncology (ESTRO) to create a complete system for the characterization and classification of oligometastatic disease based on a decision tree and a nomenclature established by a Delphi consensus. This classification aims at limiting the heterogeneity of patients enrolled in new clinical trials, exploiting an international register (E2-RADIatE platform) of real-world data (7).

Despite the heterogeneity of the population included in clinical trials, a positive trend in local disease control and OS by radical treatments in this setting is clear. The available evidence is provided by several retrospective series, phase II studies and a recently presented phase III trial.

SABR COMET is an international phase 2 study comparing Stereotactic ABlative Radiotherapy (SABR) versus standard of care systemic palliative treatment in different oligometastatic cancers with OS as the primary end-point (8). Ninety-nine patients with a controlled primary tumor and 1 to 5 metastases were randomly assigned to standard of care treatment or standard of care plus SABR to all metastatic sites. The study included 18 patients with NSCLC. SABR was associated with OS improvement [median OS 41 months (26-not reached) vs. 28 months, 95\% CI: 19-33, HR 0.57, $\mathrm{P}=0.09$ ] and doubled median progression free survival (PFS), at the cost of increased toxicity with 3 fatal events in the SABR group.

Gomez et al. conducted a multi-institutional randomized study that evaluated Local Consolidative Therapy (LCT) as compared to observation/maintenance therapy in oligometastatic NSCLC patients who had completed a first line treatment. Forty-nine patients with a controlled primary tumor were randomly assigned to maintenance therapy/ observation (MT/O) or to LCT (such as radiation therapy or surgery) to all disease sites. The trial was closed early due to the significant benefit of LCT at the preplanned annual analysis (median PFS 11.9 vs. 3.9 months, $\mathrm{P}=0.005$ ). Further follow-up has shown that LCT was also associated with a lasting benefit over time in terms of median OS (41.2 vs. 17 months, $\mathrm{P}=0.017$ ). Both early and late LCT (defined as LCT given at the time of progression in patients allowed to crossover) correlated with improved OS, even if the strategy of early local treatment seems preferable as late LCT was not feasible in patients who experienced a worsening of performance status and a wide spread progression. Moreover, patients in the LCT group survived longer after progression compared to patients in the $\mathrm{MT} / \mathrm{O}$ group (37.6 vs. 9.4 months, $\mathrm{P}=0.034)(9)$.

Iyengar et al. used a similar design to carry out a singleinstitution study comparing maintenance chemotherapy alone to maintenance plus SABR in 29 patients with oligometastatic (up to 6 metastases) NSCLC. Patients had to achieve stable disease or partial response after a firstline platinum-based chemotherapy. At the interim analysis, SABR led to higher PFS (median PFS 9.7 vs. 3.5 months, $\mathrm{P}=0.01$ ) without an increase in toxicity, so the study was prematurely stopped (10).

These three randomized trials show that locoregional treatments could increase survival in patients with oligometastatic NSCLC. However, beside the already mentioned differences in inclusion criteria with regard to number of metastatic sites, all enrolled patients already derived benefits from systemic therapy. Moreover, all these trials have been conducted before the wide adoption of immune checkpoint inhibitors (ICIs) in NSCLC treatment.

Indeed, these drugs has transformed the treatment approach to metastatic and locally advanced NSCLC, leading to unprecedented survival results. ICIs activity is based on the blockade of inhibitory signals that cancer provides to immune cells. Therefore, the administration of these agents enables $\mathrm{T}$ cells to attack tumor, after specific antigens recognition. Local ablative therapies (LAT), especially radiation, may increase neo-antigens release leading to an "immune-permissive" milieu, as suggested by preclinical studies (11). This premise has led investigators to design clinical trials addressing the combination of LAT and ICIs, even in oligometastatic patients.

Bauml et al. led the first single-arm phase 2 study evaluating the role of immunotherapy after LAT in patients with synchronous or metachronous oligometastatic NSCLC, excluding those who had high disease burden and then got to an oligometastatic state after disease response to systemic therapy (defined as oligoremnant disease). They enrolled 45 patients with up to 4 metastases, who had already completed LAT to all sites of tumor. 
Pembrolizumab (ICI directed against programmed death-1 receptor, PD-1) was given to all patients regardless of their programmed death ligand-1 (PD-L1) expression or molecular status and for at least 8 doses. After a median follow-up of 25 months, the mPFS was 19.1 months, approximately three times higher than the historical control, without new safety signals. Among the 23 patients who experienced disease progression, more than a half had systemic spread that did not involve sites of LAT (12). As compared to previously discussed trial, patients were less selected, as response to systemic therapy was not mandatory for inclusion.

Another area of active interest is that of oncogeneaddicted NSCLC. Most of the aforementioned randomized trials did not include this population or just few cases as in the case of the study by Gomez et al., that enrolled 8 patients with epidermal growth factor receptor (EGFR) mutations or anaplastic lymphoma kinase (ALK) rearrangements (9).

$\mathrm{Xu}$ and colleagues conducted the first large-scale retrospective study to investigate whether consolidative LAT can improve survival of patients with oligometastatic EGFR-mutant NSCLC (up to 5 metastases) responding to a first-line EGFR tyrosine kinase inhibitors (TKI) therapy. According to the propensity score matching analysis, consolidation treatment with radiotherapy and/or surgery administered to all disease sites resulted in an improvement in OS (mOS 39.7 vs. 24.8 months, HR 0.44, 95\% CI: 0.27-0.71, $\mathrm{P}=0.001$ ) and PFS (mPFS 20.1 vs. 11.9 months, HR $0.38,95 \%$ CI: $0.24-0.62, \mathrm{P}<0.001)$ compared to partial treatment on a site of disease or to observation (13).

The optimal sequence of administration of local treatments and target therapies in NSCLC patients with driving mutations is controversial. In a multi-institutional retrospective analysis, upfront Stereotactic Radio Surgery (SRS) followed by EGFR-TKI in patients with baseline brain metastases led to a longer OS compared both with whole brain radiation therapy (WBRT), followed by EGFR-TKI and upfront EGFR-TKI, followed by deferred radiotherapy (14). However, such data must be put into the current clinical arena, where novel TKIs with greater central nervous system penetration and activity have undermined old generation drugs both in EGFR and ALK positive patients.

Recently, Peng et al. started a randomized, multicenter phase 2 study comparing TKI therapy plus SBRT to TKI alone in 61 EGFR positive patients affected by oligometastatic NSCLC (up to 3 metastatic sites). SBRT was administered 3 months after the start of systemic therapy in patients that achieved partial response or stable disease. A trend towards improved PFS was observed in patients receiving SBRT for primary tumor combined with EGFR TKI (17.4 vs. 8.9 months, $\mathrm{P}=0.042$ ). Despite the continuation of the TKI throughout SBRT, neither toxicity nor the incidence of grade $\geq 3$ adverse events increased in the combination arm. In addition, the administration of the combined treatment seems to delay the appearance of mechanisms of resistance to TKIs, with a benefit in terms of PFS (mPFS 17.4 vs. 10.3 months, $\mathrm{P}=0.007$ ) (15). The molecular mechanism for this association is unclear, even if we can speculate that SBRT may have reduced the number of resistant clones, thus delaying their emergence.

At 2020 American Society of Clinical Oncology (ASCO) Virtual Annual Meeting, Wang presented interim results of the phase III SINDAS study, an open-label, randomized trial comparing upfront SBRT plus TKI and TKI alone in EGFR positive advanced NSCLC (16). Patients were treated with old generation TKI (gefitinib, erlotinib, or icotinib) and had no more than 5 total metastases with a maximum of 2 lesions in each involved organ. Primary endpoint was PFS. The study enrolled 136 patients, mostly with common EGFR mutations even if $4 \%$ and $12 \%$ in the experimental and control arm, respectively, had exon 20 insertions, often resistant to TKIs. Upfront SBRT plus EGFR TKI significantly improved PFS (HR 0.618, 95\% CI: $0.394-0.969, \mathrm{P}<0.001$ ) and led to superior OS although the difference was not statistically significant. No significant differences in terms of toxicity were reported.

Beside radiation therapy, surgery has a clear role in oligometastatic patients too. A large retrospective study on patients with stage IV NSCLC who underwent surgery to both the primary tumor and metastatic sites showed that combined surgery gave an overall and a cancer-related survival benefit, both before and after propensity score matching. Subgroup analysis suggests that surgery could be effective especially in patients with single brain metastases or contralateral pulmonary nodules (17). Although available data are still scarce, phase 3 trials are ongoing.

The phase 3 OMEGA trial (NCT03827577) is recruiting and randomizing patients with oligometastatic NSCLC to standard treatment plus local ablative therapy (surgery and/ or radiotherapy) or to standard treatment alone. Patients are stratified according to synchronous or metachronous presentation, number of metastases, nodal status, oncogeneaddiction and PD-L1 expression levels. Similarly, the multicenter randomized phase 3 SARON trial (NCT02417662) will assess efficacy and safety of SABR in addition to standard 
chemotherapy compared to standard treatment alone in the oligometastatic EGFR/ALK negative patients with similar stratification factors (18). Conversely, the HALT phase 3 trial (NCT03256981) is exploring if SBRT plus TKI improves PFS as compared to TKI alone beyond oligo-progression in patients with oncogene-driven NSCLC.

Finally, the OITROLC phase 3 trial (NCT02076477) is underway to define the optimal timing for the administration of radiation therapy, by randomizing patients between upfront chemo plus concurrent radiotherapy to the primary and all metastatic sites versus a consolidative approach after two cycles of induction chemotherapy.

In conclusion, available data suggest that oligometastatic NSCLC patients may achieve long term disease control and, sometimes, even cure. For this reason, waiting for the results of these trials, the current approach to oligometastatic NSCLC patients should be based on a single case discussion in experienced multidisciplinary tumor boards, considering both the disease characteristics, the local expertise and, last but not least, patient preferences.

The wide adoption of stringent and shared definitions such as those proposed by the EORTC-LCG is warranted to daily practice as well as to harmonize inclusion criteria and staging procedures for future clinical trials (6). Efforts such as the OligoCare registry would add further realworld data to strengthen evidences (7). The evolution of the treatment landscape in NSCLC, both in the advanced and early stages, proposes continuous new challenges. As already mentioned, the introduction of ICIs is putting us into unexplored landscapes, where the potential efficacy of LAT in combination with an awakened immune system clearly deserves well designed clinical trials. Further on, novel molecular targets are emerging along with specific molecules, rapidly expanding the field of precision oncology. The future of oligometastatic disease patient management must therefore incorporate evidence-based approaches along with continuous cultural exchange between specialists to finally get to real personalized medicine.

\section{Acknowledgments}

Funding: None.

\section{Footnote}

Provenance and Peer Review: This article was commissioned by the Guest Editors (Maurizio Infante \& Thierry Berghmans) for the series "Oligometastatic NSCLC: definition and treatment opportunities" published in Translational Lung Cancer Research. The article did not undergo external peer review.

Conflicts of Interest: All authors have completed the ICMJE uniform disclosure form (available at http://dx.doi. org/10.21037/tlcr-20-771). The series "Oligometastatic NSCLC: definition and treatment opportunities" was commissioned by the editorial office without any funding or sponsorship. SN serves as an unpaid editorial board member of Translational Lung Cancer Research from July 2019 to July 2021. SN reports conflict of interest as advisor, speaker bureau: AZ, BI, MSD, Roche, Takeda, Pfizer, Beigene, Sanofi, Novartis, BMS, Eli Lilly. The authors have no other conflicts of interest to declare.

Ethical Statement: The authors are accountable for all aspects of the work in ensuring that questions related to the accuracy or integrity of any part of the work are appropriately investigated and resolved.

Open Access Statement: This is an Open Access article distributed in accordance with the Creative Commons Attribution-NonCommercial-NoDerivs 4.0 International License (CC BY-NC-ND 4.0), which permits the noncommercial replication and distribution of the article with the strict proviso that no changes or edits are made and the original work is properly cited (including links to both the formal publication through the relevant DOI and the license). See: https://creativecommons.org/licenses/by-nc-nd/4.0/.

\section{References}

1. Hellman S, Weichselbaum RR. Oligometastases. J Clin Oncol 1995;13:8-10.

2. Eberhardt WE, Mitchell A, Crowley J, et al. The IASLC Lung Cancer Staging Project: proposals for the revision of the $M$ descriptors in the forthcoming eighth edition of the TNM classification of lung cancer. J Thorac Oncol 2015;10:1515-22.

3. Planchard D, Popat S, Kerr K, et al. Metastatic non-small cell lung cancer: ESMO Clinical Practice Guidelines for diagnosis, treatment and follow-up. Ann Oncol 2018;29:iv192-iv237.

4. Novello S, Barlesi F, Califano R, et al. Metastatic nonsmall-cell lung cancer: ESMO Clinical Practice Guidelines for diagnosis, treatment and follow-up. Ann Oncol 2016;27:v1-v27. 
5. Levy A, Hendriks LEL, Berghmans T, et al. EORTC Lung Cancer Group survey on the definition of NSCLC synchronous oligometastatic disease. Eur J Cancer 2019;122:109-14.

6. Dingemans AC, Hendriks LEL, Berghmans T, et al. Definition of Synchronous Oligometastatic Non-Small Cell Lung Cancer-A Consensus Report. J Thorac Oncol 2019;14:2109-19.

7. Guckenberger $M$, Lievens $Y$, Bouma AB, et al. Characterisation and classification of oligometastatic disease: a European Society for Radiotherapy and Oncology and European Organisation for Research and Treatment of Cancer consensus recommendation. Lancet Oncol 2020;21:e18-e28.

8. Palma DA, Olson R, Harrow S, et al. Stereotactic ablative radiotherapy versus standard of care palliative treatment in patients with oligometastatic cancers (SABRCOMET): a randomised, phase 2, open-label trial. Lancet 2019;393:2051-8.

9. Gomez DR, Tang C, Zhang J, et al. Local Consolidative Therapy Vs. Maintenance Therapy or Observation for Patients With Oligometastatic Non-Small-Cell Lung Cancer: Long-Term Results of a Multi-Institutional, Phase II, Randomized Study. J Clin Oncol 2019;37:1558-65.

10. Iyengar P, Wardak Z, Gerber DE, et al. Consolidative Radiotherapy for Limited Metastatic Non-Small-Cell Lung Cancer: A Phase 2 Randomized Clinical Trial. JAMA Oncol 2018;4:e173501.

11. Sharabi AB, Lim M, DeWeese TL, et al. Radiation and checkpoint blockade immunotherapy: radiosensitisation and potential mechanisms of synergy. Lancet Oncol 2015;16:e498-e509.

12. Bauml JM, Mick R, Ciunci C, et al. Pembrolizumab

Cite this article as: Bungaro $\mathrm{M}$, Paratore C, Bironzo $\mathrm{P}$, Novello S. Local for advanced, is this a paradox? Transl Lung Cancer Res 2021;10(7):3324-3328. doi: 10.21037/tlcr-20-771
After Completion of Locally Ablative Therapy for Oligometastatic Non-Small Cell Lung Cancer: A Phase 2 Trial. JAMA Oncol 2019;5:1283-90.

13. Xu Q, Zhou F, Liu H, et al. Consolidative Local Ablative Therapy Improves the Survival of Patients With Synchronous Oligometastatic NSCLC Harboring EGFR Activating Mutation Treated With First-Line EGFRTKIs. J Thorac Oncol 2018;13:1383-92.

14. Magnuson WJ, Lester-Coll NH, Wu AJ, et al. Management of Brain Metastases in Tyrosine Kinase Inhibitor-Naïve Epidermal Growth Factor ReceptorMutant Non-Small-Cell Lung Cancer: A Retrospective Multi-Institutional Analysis. J Clin Oncol 2017;35:1070-7.

15. Peng P, Chen Y, Han G, et al. Concomitant SBRT and EGFR-TKI Versus EGFR-TKI Alone for Oligometastatic NSCLC: A Multicenter, Randomized Phase II Study. J Thorac Oncol 2019;14:S250-1.

16. Wang $X$, First-line tyrosine kinase inhibitor with or without aggressive upfront local radiation therapy in patients with EGFRm oligometastatic non-small cell lung cancer: interim results of a randomized phase III, openlabel clinical trial (SINDAS) (NCT02893332. J Clin Oncol 2020;38:abstr 9508.

17. Abdel-Rahman O. Outcomes of Surgery as Part of the Management of Metastatic Non-Small-Cell Lung Cancer: A Surveillance, Epidemiology and End Results Database Analysis. Cancer Invest 2018;36:238-45.

18. Conibear J, Chia B, Ngai Y, et al. Study protocol for the SARON trial: a multicentre, randomised controlled phase III trial comparing the addition of stereotactic ablative radiotherapy and radical radiotherapy with standard chemotherapy alone for oligometastatic non-small cell lung cancer. BMJ Open 2018;8:e020690. 